\title{
A total-variation surface energy model for thin films of martensitic crystals
}

\author{
PAVEL BĚLÍK ${ }^{\dagger}$ AND MITCHELL LUSKIN $\$ \S$ \\ School of Mathematics, University of Minnesota, 206 Church Street SE, Minneapolis, \\ MN 55455, USA
}

[Received 2 April 2001]

\begin{abstract}
We rigorously derive a thin-film limit for martensitic crystals that utilizes the total variation of the deformation gradient to model the energy on surfaces separating regions of different variants. We find that the deformation for an infinitesimally thin film minimizes a two-dimensional energy.
\end{abstract}

Keywords: Thin film; surface energy; bounded variation; martensite

\section{Introduction}

Martensitic thin films have applications in actuators, sensors, and micromachines because of their large work output/(cycle · volume) [22]. Single-crystal martensitic thin films have recently been grown in the laboratory [12] and theoretically offer even larger work output/(cycle $\cdot$ volume $)$ [5].

Bhattacharya and James [5] have rigorously derived a thin-film variational principle from the three-dimensional elastic energy for martensite with the surface energy modeled by $\kappa \int_{\Omega_{h}}\left|\nabla^{2} u\right|^{2} \mathrm{~d} x$, where $\kappa$ is a small positive strain-gradient coefficient, $\Omega_{h}$ is the reference configuration of a crystal with thickness $h$, and $\int_{\Omega_{h}}\left|\nabla^{2} u\right|^{2} \mathrm{~d} x$ is the square of the $L^{2}$-norm of the matrix of all the second derivatives of the deformation $u$. Unless we set $\kappa=0$, deformations with finite energy for this thinfilm model cannot have sharp interfaces between two compatible variants of martensite or between austenite and martensite.

Our total-variation model allows the use of continuous, piecewise linear approximations of the deformation. Conforming numerical approximations of the Bhattacharya-James thin-film energy require higher-order finite-element approximations [8]. We also note that although mixed finiteelement methods can be used to approximate a plate problem (and presumably the BhattacharyaJames thin-film model) with piecewise linear deformations, the mixed variational formulation transforms the primal energy-minimization problem to a more computationally challenging saddlepoint problem [11].

For this reason, we give a derivation of an alternative thin-film variational principle in which the interfacial energy is modeled by a term of the form $\kappa \int_{\Omega_{h}}|D(\nabla u)|$, where $\kappa$ again denotes a small positive strain-gradient coefficient and $\int_{\Omega_{h}}|D(\nabla u)|$ denotes the total variation of the deformation gradient $\nabla u$ in $\Omega_{h}$. Deformations of finite energy can have sharp interfaces with this model, and it can be seen that the interfacial energy is concentrated along the surfaces separating regions of constant deformation gradient. Hence, when continuous, piecewise linear finite elements are

\footnotetext{
Email: belik@math.umn.edu

*Email: luskin@math.umn.edu

${ }^{\S}$ Corresponding author
} 
used for numerical simulations, the interfacial energy is concentrated along the edges of the finite element triangulation. Not only does this property make this model computationally attractive, but we believe it also better models deformations for martensitic crystals with small surface energy. We have successfully used our total variation model to compute a stress-induced martensitic phase transformation of a single-crystal thin film by indentation and its reverse transformation to austenite by heating [10].

A similar approach to the interfacial energy has been studied in [17] and a rigorous analysis of the relation between the two models for the interfacial energy has been given in [19-21] for some scalar models. See also [4: p. 44] and [28] for arguments relating the surface energy to the fineness of microstructures in martensitic crystals. We have proven the ' $\Gamma$-convergence' of the rescaled threedimensional energy to a two-dimensional energy. Related work can be found in [1, 3, 13, 16, 26, 27].

In Section 2, we describe the three-dimensional model with the total-variation interfacial energy for thin films with finite thickness. In Section 3, we present some known properties of functions of bounded variation and prove two lemmas needed for the derivation of the thin film-theory. Using these results for functions of bounded variation, we show in Section 4 by using the direct method of the calculus of variations that for any positive thickness of the film there exists a minimizer of the bulk energy (2.5). Next, in Section 5, we analyze the behavior of the minimizers as the thickness of the film tends to zero. We show that there exists a convergent subsequence of these minimizers, a limiting two-dimensional energy, and a two-dimensional minimum principle allowing one to characterize and numerically compute the limiting deformations (Theorem 5.1).

Finally, in Section 6, we briefly describe the finite-element approximation of the model derived in Section 2. We give the exact expression for the thin-film energy for continuous, piecewise linear deformations. More details about the computer implementation and the results of numerical simulations will appear in a subsequent paper.

\section{The thin film of finite thickness}

We will assume that $S \subset \mathbb{R}^{2}$ is a bounded domain (connected, open set) with a Lipschitz continuous boundary and denote the reference undistorted configuration of the thin film of the martensitic material by $\Omega_{h}, 0<h \leqslant 1$, where

$$
\Omega_{h}=S \times(-h / 2, h / 2) .
$$

In what follows, we will consider functions whose domain is $\Omega_{h}$ and whose range is $\mathbb{R}^{3}$. The gradient of a typical such function $\tilde{u}$ belongs to $\mathbb{R}^{3 \times 3}$ and will be denoted by $\nabla \tilde{u}$. We use the notation $\tilde{u}_{i, j}=\partial \tilde{u}_{i} / \partial x_{j}$, and we denote the columns of $\nabla \tilde{u}$ by $\tilde{u}_{, i}, i=1,2,3$.

We denote by $\left(V_{1} \mid V_{2}\right) \in \mathbb{R}^{3 \times 2}$ the matrix whose columns are $V_{1}, V_{2} \in \mathbb{R}^{3}$. The 'planar' gradient of $\tilde{u}$ can then be denoted by $\nabla_{P} \tilde{u}=\left(\tilde{u}_{1,1} \mid \tilde{u}_{, 2}\right) \in \mathbb{R}^{3 \times 2}$. We shall use this notation more generally, so we will denote by $\left(V_{12} \mid V_{3}\right) \in \mathbb{R}^{3 \times 3}$ the matrix whose first two columns are given by $V_{12} \in \mathbb{R}^{3 \times 2}$ and whose third column is given by $V_{3} \in \mathbb{R}^{3}$, and we will denote by $\left(V_{12}\left|V_{3}\right| V_{4}\right) \in \mathbb{R}^{3 \times 4}$ the matrix whose first two columns are given by $V_{12} \in \mathbb{R}^{3 \times 2}$ and whose third and fourth columns are given by $V_{3}, V_{4} \in \mathbb{R}^{3}$. by

Given an open set $\Omega \subset \mathbb{R}^{n}$ and a function $v \in L^{1}(\Omega ; \mathbb{R})$, we define the total variation of $v$ [18]

$$
\int_{\Omega}|D v|=\sup \left\{\int_{\Omega} v(x) \operatorname{div} \psi(x) \mathrm{d} x: \psi \in \mathcal{C}_{0}^{\infty}\left(\Omega ; \mathbb{R}^{n}\right),|\psi(x)| \leqslant 1 \text { for all } x \in \Omega\right\}
$$


and say $v \in B V(\Omega)$ if $\int_{\Omega}|D v|<+\infty$. We recall that

$$
\mathcal{C}_{0}^{\infty}\left(\Omega ; \mathbb{R}^{n}\right)=\left\{\psi \in \mathcal{C}^{\infty}\left(\Omega ; \mathbb{R}^{n}\right): \operatorname{supp} \psi \text { is a compact subset of } \Omega\right\} .
$$

In what follows, we sometimes suppress the target space of $\psi$ from the notation and simply write $\psi \in \mathcal{C}_{0}^{\infty}(\Omega)$ with the corresponding space tacitly assumed. For a matrix-valued function $v \in L^{1}\left(\Omega ; \mathbb{R}^{m \times p}\right)$, we define

$\int_{\Omega}|D v|=\sup \left\{\sum_{\substack{i=1, \ldots, m \\ j=1, \ldots, p}} \int_{\Omega} v_{i j}(x) \operatorname{div} \psi_{i j}(x) \mathrm{d} x: \psi \in \mathcal{C}_{0}^{\infty}\left(\Omega ; \mathbb{R}^{m \times p \times n}\right),|\psi(x)| \leqslant 1\right.$ for all $\left.x \in \Omega\right\}$

and say $v \in B V(\Omega)$ if $\int_{\Omega}|D v|<+\infty$. Here and in what follows, the vector norm $|\cdot|$ is the usual Euclidean norm, that is, the square root of the sum of the squares of all the components. Finally, we define the 'planar' variation

$\int_{\Omega}\left|D_{P} v\right|=\sup \left\{\sum_{\substack{i=1, \ldots, m \\ j=1, \ldots, p \\ k=1,2}} \int_{\Omega} v_{i j}(x) \psi_{i j k, k}(x) \mathrm{d} x: \psi \in \mathcal{C}_{0}^{\infty}\left(\Omega ; \mathbb{R}^{m \times p \times 2}\right),|\psi(x)| \leqslant 1\right.$ for all $\left.x \in \Omega\right\}$ and remark that if $v \in B V\left(\Omega_{1}\right)$ is independent of $x_{3}$, then, abusing the notation slightly, we have

$$
\int_{\Omega_{1}}|D v|=\int_{\Omega_{1}}\left|D_{P} v\right|=\int_{S}|D v| .
$$

Next, we will assume that the energy density $\phi: \mathbb{R}^{3 \times 3} \times\left(\theta_{0}, \theta_{1}\right) \rightarrow[0,+\infty)$ is a continuous function satisfying the growth condition

$$
c_{1}|F|^{p}-c_{2} \leqslant \phi(F, \theta) \leqslant c_{3}\left(|F|^{p}+1\right) \quad \text { for all } F \in \mathbb{R}^{3 \times 3} \text { and } \theta \in\left(\theta_{0}, \theta_{1}\right),
$$

where $c_{1}, c_{2}$, and $c_{3}$ are fixed positive constants, $\theta_{0}<\theta_{1}$ represent a range of temperature, and $3<p<+\infty$. The function $\phi$ represents the energy density for martensitic crystals, but, other than the properties mentioned above, we do not impose any additional assumptions on it. The two arguments of $\phi$ are the deformation gradient $F$ and the temperature $\theta$.

We assume that the film adheres to a rigid material on the surface

$$
\Gamma_{h}=\gamma \times(-h / 2, h / 2),
$$

where we assume that $\gamma \neq \emptyset$ is a finite union of connected $\mathcal{C}^{1,1}$ open subsets of $\partial S$. Let $y_{0}, b_{0} \in$ $W^{1, p}\left(S ; \mathbb{R}^{3}\right)$ be such that $\nabla y_{0}, \nabla b_{0} \in B V(S)$ and define

$$
\tilde{u}_{0}\left(x_{1}, x_{2}, x_{3}\right)=y_{0}\left(x_{1}, x_{2}\right)+b_{0}\left(x_{1}, x_{2}\right) x_{3} \quad \text { for }\left(x_{1}, x_{2}, x_{3}\right) \in \Omega_{h} .
$$

We then define the space $\mathcal{A}_{h}$ of admissible deformations of the domain $\Omega_{h}$ by

$$
\mathcal{A}_{h}=\left\{\tilde{u} \in W^{1, p}\left(\Omega_{h} ; \mathbb{R}^{3}\right): \nabla \tilde{u} \in B V\left(\Omega_{h}\right), \tilde{u}=\tilde{u}_{0} \text { on } \Gamma_{h}\right\} .
$$


If the crystal adheres to a part of the crystal that is constrained by a substrate to be in the austenitic phase, then we can model the boundary constraint simply [5] by

$$
\tilde{u}_{0}\left(x_{1}, x_{2}, x_{3}\right)=\left(x_{1}, x_{2}, x_{3}\right) \text { for }\left(x_{1}, x_{2}, x_{3}\right) \in \Omega_{h}
$$

so that

$$
\left.\begin{array}{l}
y_{0}\left(x_{1}, x_{2}\right)=\left(x_{1}, x_{2}, 0\right) \\
b_{0}\left(x_{1}, x_{2}\right)=(0,0,1)
\end{array}\right\} \quad \text { for }\left(x_{1}, x_{2}, x_{3}\right) \in \Omega_{h} .
$$

We are interested in computing energy-minimizing deformations for asymptotically thin films with a fixed (measurable) distribution of the temperature $\theta(x) \in\left(\theta_{0}, \theta_{1}\right)$, so we will present results for the behavior of the minimizers $\tilde{u}_{h}$ over the spaces $\mathcal{A}_{h}$ of the energy

$$
\mathcal{E}_{h}(\tilde{u}, \theta)=\kappa \int_{\Omega_{h}}|D(\nabla \tilde{u})|+\int_{\Omega_{h}} \phi(\nabla \tilde{u}(x), \theta(x)) \mathrm{d} x
$$

for fixed $\kappa>0$ as $h \rightarrow 0$. Since the temperature distribution will be held fixed in the derivation, we suppress it from the notation and use $\mathcal{E}_{h}(\tilde{u})$ to stand for $\mathcal{E}_{h}(\tilde{u}, \theta)$.

REMARK 2.1 Note that due to the growth condition (2.2), we have

$$
\mathcal{A}_{h}=\left\{\tilde{u}: \Omega_{h} \rightarrow \mathbb{R}^{3}: \mathcal{E}_{h}(\tilde{u})<+\infty, \tilde{u}=\tilde{u}_{0} \text { on } \Gamma_{h}\right\} .
$$

Also, since $p>3$, it follows from the Sobolev embedding theorem [2] that $\mathcal{A}_{h} \subset \mathcal{C}\left(\bar{\Omega}_{h}\right)$. This ensures that there is no tear in the deformed configurations $\tilde{u}\left(\Omega_{h}\right)$ for $\tilde{u} \in \mathcal{A}_{h}$.

We will prove in Theorem 5.1 in Section 5 that energy-minimizing deformations $\tilde{u}_{h}$ for the energy (2.5) for thin films of finite thickness can be approximated as $h \rightarrow 0$ by deformations

$$
y\left(x_{1}, x_{2}\right)+b\left(x_{1}, x_{2}\right) x_{3} \quad \text { for }\left(x_{1}, x_{2}, x_{3}\right) \in \Omega_{h}=S \times(-h / 2, h / 2)
$$

such that $(y, b)$ minimizes the thin-film energy

$$
\mathcal{E}^{(0)}(y, b)=\kappa\left[\int_{S}|D(\nabla y|b| b)|+\sqrt{2} \int_{\gamma}\left|b-b_{0}\right|\right]+\int_{S} \phi(\nabla y \mid b)
$$

in the space $\mathcal{A}_{0}$ of admissible deformations of the domain $S$ given by

$$
\mathcal{A}_{0}=\left\{(y, b) \in W^{1, p}\left(S ; \mathbb{R}^{3}\right) \times L^{p}\left(S ; \mathbb{R}^{3}\right): \nabla y, b \in B V(S), y=y_{0} \text { on } \gamma\right\} .
$$

We will show in Section 6 that the thin-film energy (2.6) has a very simple form when restricted to continuous, piecewise linear $y$ and piecewise constant $b$.

\section{Properties of functions of bounded variation}

Before we proceed with the derivation of the thin-film theory, we recall and prove extensions of some results about functions of bounded variation that will be useful in what follows. Variants of these results can be found, for example, in $[15,18]$. 
THEOREM 3.1 (Semi-continuity) If $\left\{v_{j}\right\}$ is a sequence of functions which converge in $L_{\text {loc }}^{1}(\Omega)$ to a function $v$, then

$$
\int_{\Omega}|D v| \leqslant \liminf _{j \rightarrow \infty} \int_{\Omega}\left|D v_{j}\right| .
$$

Now let $S$ be a domain in $\mathbb{R}^{2}$ and $\Omega_{1}=S \times(-1 / 2,1 / 2)$. If $\left\{v_{j}\right\}$ is a sequence of functions which converge in $L_{\text {loc }}^{1}\left(\Omega_{1}\right)$ to a function $v$, then

$$
\int_{\Omega_{1}}\left|D_{P} v\right| \leqslant \liminf _{j \rightarrow \infty} \int_{\Omega_{1}}\left|D_{P} v_{j}\right| \text {. }
$$

LEMMA 3.1 The space $B V(\Omega)$ is a Banach space with respect to the norm

$$
\|v\|_{B V(\Omega)}=\|v\|_{L^{1}(\Omega)}+\int_{\Omega}|D v| .
$$

THEOREM 3.2 (Compactness) Let $\Omega$ be a bounded open set in $\mathbb{R}^{n}$ with a Lipschitz continuous boundary $\partial \Omega$. Then bounded subsets of $B V(\Omega)$ are relatively compact in $L^{1}(\Omega)$.

By using the construction and a slight extension of the proof of Theorem 1.17 in [18], we have the following theorem.

THEOREM 3.3 (Approximation of functions in $B V(\Omega)$ ) Let $1 \leqslant q<+\infty$ and let $\Omega$ be a bounded open set in $\mathbb{R}^{n}$ with a Lipschitz continuous boundary, $\partial \Omega$. For every $v \in L^{q}(\Omega) \cap B V(\Omega)$, there exists a sequence $\left\{v_{j}\right\} \subset \mathcal{C}^{\infty}(\Omega)$ such that $v_{j}=v$ on $\partial \Omega$ and such that

$$
\begin{aligned}
& \lim _{j \rightarrow \infty}\left\|v_{j}-v\right\|_{L^{q}(\Omega)}=0, \\
& \lim _{j \rightarrow \infty} \int_{\Omega}\left|D v_{j}\right|=\int_{\Omega}|D v| .
\end{aligned}
$$

Furthermore, if $\nabla v \in B V(\Omega)$, we may construct the $v_{j}$ above so that $\nabla v_{j} \in B V(\Omega)$.

If $\Omega_{1}=S \times(-1 / 2,1 / 2)$ where $S$ is a domain in $\mathbb{R}^{2}$ with a Lipschitz continuous boundary, $\partial S$, then for every $v \in L^{q}\left(\Omega_{1}\right) \cap B V\left(\Omega_{1}\right)$, there exists a sequence $\left\{v_{j}\right\} \subset \mathcal{C}^{\infty}\left(\Omega_{1}\right)$ such that $v_{j}=v$ on $\partial S \times(-1 / 2,1 / 2)$ and such that

$$
\begin{gathered}
\lim _{j \rightarrow \infty}\left\|v_{j}-v\right\|_{L^{q}\left(\Omega_{1}\right)}=0, \\
\lim _{j \rightarrow \infty} \int_{\Omega_{1}}\left|D_{P} v_{j}\right|=\int_{\Omega_{1}}\left|D_{P} v\right| .
\end{gathered}
$$

We will also need the following two lemmas which refine a special case of the results of Theorems 3.1 and 3.3.

LEMmA 3.2 (Semi-continuity) Let $S$ be a bounded domain in $\mathbb{R}^{2}$ with a Lipschitz continuous boundary, $\partial S$, and let $\gamma \neq \emptyset$ be a finite union of connected $\mathcal{C}^{1,1}$ open subsets of $\partial S$. If $w_{j}, b_{j}$ and $w, b$ are functions in $B V\left(\Omega_{1}\right)$ satisfying $b_{j}=b_{0}$ on $\Gamma_{1}=\gamma \times(-1 / 2,1 / 2)$ for fixed $b_{0} \in B V\left(\Omega_{1}\right)$ and

$$
\lim _{j \rightarrow \infty}\left\|w_{j}-w\right\|_{L^{1}\left(\Omega_{1}\right)}=0 \quad \text { and } \quad \lim _{j \rightarrow \infty}\left\|b_{j}-b\right\|_{L^{1}\left(\Omega_{1}\right)}=0
$$

then

$$
\int_{\Omega_{1}}\left|D_{P}(w \mid b)\right|+\int_{\Gamma_{1}}\left|b-b_{0}\right| \leqslant \liminf _{j \rightarrow \infty} \int_{\Omega_{1}}\left|D_{P}\left(w_{j} \mid b_{j}\right)\right| .
$$




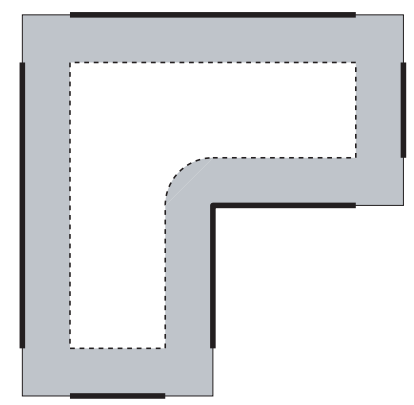

FIG. 1. An example of the sets used in the proof of Lemma 3.2. The shaded area is a horizontal section $x_{3}=c$ of $\Omega^{(\delta)}$, the dashed line denotes $\Gamma^{(\delta)}$, and the union of the thick lines denotes $\Gamma_{\partial}^{(\delta)}$.

Proof. Let $n(x)$ denote the inward-pointing normal vector at $x \in \Gamma_{1}$. For $\delta>0$, we define the following sets (see Fig. 1 for an example):

$$
\begin{aligned}
& \Gamma^{(\delta)}=\left\{x \in \Omega_{1}: \operatorname{dist}\left(x, \Gamma_{1}\right)=\delta\right\}, \quad \Gamma_{\partial}^{(\delta)}=\left\{x \in \Gamma_{1}: x+\delta n(x) \in \Gamma^{(\delta)}\right\}, \\
& \Omega^{(\delta)}=\left\{x \in \Omega_{1}: \operatorname{dist}(x, \partial S \times(-1 / 2,1 / 2))<\delta\right\} .
\end{aligned}
$$

Given sufficiently small $\delta>0$, we have for $0<v<\delta$ and $\zeta \in \mathcal{C}^{\infty}\left(\Omega_{1}\right) \cap B V\left(\Omega_{1}\right)$ the identity

$$
\zeta(x)=-\int_{0}^{\nu} D \zeta(x+\sigma n(x)) \cdot n(x) \mathrm{d} \sigma+\zeta(x+\nu n(x)) \quad \text { for } x \in \Gamma_{\partial}^{(\delta)},
$$

so we can derive the trace inequality

$$
\int_{\Gamma_{\partial}^{(\delta)}}|\zeta| \leqslant(1-L \delta)^{-1}\left(\int_{\Omega^{(\delta)}}\left|D_{P} \zeta\right|+\delta^{-1} \int_{\Omega^{(\delta)}}|\zeta|\right) \quad \text { for } \zeta \in \mathcal{C}^{\infty}\left(\Omega_{1}\right) \cap B V\left(\Omega_{1}\right)
$$

where $L$ denotes the maximum of the Lipschitz constants for $n(x)$ since the Jacobian of the inverse of the map $(x, \sigma) \in \Gamma_{\partial}^{(\delta)} \times(0, \delta) \mapsto x+\sigma n(x) \in \Omega^{(\delta)}$ is bounded above by $(1-L \delta)^{-1}$. It follows from Theorem 3.3 that (3.1) holds for $\zeta \in B V\left(\Omega_{1}\right)$. We can then set $\zeta=(w \mid b)-\left(w_{j} \mid b_{j}\right)$ in $(3.1)$ to obtain since $b_{j}=b_{0}$ on $\Gamma_{1}$ that

$$
\begin{gathered}
\int_{\Gamma_{\partial}^{(\delta)}}\left|b-b_{0}\right| \leqslant(1-L \delta)^{-1}\left(\int_{\Omega^{(\delta)}}\left|D_{P}\left[(w \mid b)-\left(w_{j} \mid b_{j}\right)\right]\right|+\delta^{-1} \int_{\Omega^{(\delta)}}\left|(w \mid b)-\left(w_{j} \mid b_{j}\right)\right|\right) \\
\leqslant(1-L \delta)^{-1}\left(\int_{\Omega^{(\delta)}}\left|D_{P}(w \mid b)\right|+\int_{\Omega^{(\delta)}}\left|D_{P}\left(w_{j} \mid b_{j}\right)\right|+\delta^{-1} \int_{\Omega^{(\delta)}}\left|(w \mid b)-\left(w_{j} \mid b_{j}\right)\right|\right) .
\end{gathered}
$$

For arbitrary $\varepsilon>0$, we now choose $\delta>0$ sufficiently small so that

$$
L \delta \leqslant \varepsilon, \quad \int_{\Omega^{(\delta)}}\left|D_{P}(w \mid b)\right| \leqslant \varepsilon, \quad \int_{\Gamma_{1} \backslash \Gamma_{\partial}^{(\delta)}}\left|b-b_{0}\right| \leqslant \varepsilon .
$$

We then have by (3.2) since $\left(w_{j} \mid b_{j}\right) \rightarrow(w \mid b)$ in $L^{1}\left(\Omega_{1}\right)$ that

$$
\liminf _{j \rightarrow \infty} \int_{\Omega^{(\delta)}}\left|D_{P}\left(w_{j} \mid b_{j}\right)\right| \geqslant(1-\varepsilon) \int_{\Gamma_{1}}\left|b-b_{0}\right|-\varepsilon .
$$


Using the semi-continuity Theorem 3.1 applied to $\Omega_{1} \backslash \bar{\Omega}^{(\delta)}$ and (3.3), we find that

$$
\liminf _{j \rightarrow \infty} \int_{\Omega_{1} \backslash \bar{\Omega}^{(\delta)}}\left|D_{P}\left(w_{j} \mid b_{j}\right)\right| \geqslant \int_{\Omega_{1} \backslash \bar{\Omega}^{(\delta)}}\left|D_{P}(w \mid b)\right| \geqslant \int_{\Omega_{1}}\left|D_{P}(w \mid b)\right|-\varepsilon .
$$

We thus obtain from summing (3.4) and (3.5) that

$$
\liminf _{j \rightarrow \infty} \int_{\Omega_{1}}\left|D_{P}\left(w_{j} \mid b_{j}\right)\right| \geqslant \int_{\Omega_{1}}\left|D_{P}(w \mid b)\right|+(1-\varepsilon) \int_{\Gamma_{1}}\left|b-b_{0}\right|-2 \varepsilon .
$$

The proof of the lemma follows since $\varepsilon>0$ was arbitrary.

The following corollary of Lemma 3.2 will be used in the proof of the thin film-limit. We note that

$$
\int_{\Omega_{1}}\left|D_{P}(w, b, b)\right|=\int_{\Omega_{1}}\left|D_{P}(w, \sqrt{2} b)\right| .
$$

COROLLARY 3.1 Let $S$ be a bounded domain in $\mathbb{R}^{2}$ with a Lipschitz continuous boundary, $\partial S$, and let $\gamma \neq \emptyset$ be a finite union of connected $\mathcal{C}^{1,1}$ open subsets of $\partial S$. If $w_{j}, b_{j}$ and $w, b$ are functions in $B V\left(\Omega_{1}\right)$ satisfying

$$
\lim _{j \rightarrow \infty}\left\|w_{j}-w\right\|_{L^{1}\left(\Omega_{1}\right)}=0 \quad \text { and } \quad \lim _{j \rightarrow \infty}\left\|b_{j}-b\right\|_{L^{1}\left(\Omega_{1}\right)}=0,
$$

and $b_{j}=b_{0}$ on $\Gamma_{1}=\gamma \times(-1 / 2,1 / 2)$ for fixed $b_{0} \in B V\left(\Omega_{1}\right)$, then

$$
\int_{\Omega_{1}}\left|D_{P}(w|b| b)\right|+\sqrt{2} \int_{\Gamma_{1}}\left|b-b_{0}\right| \leqslant \liminf _{j \rightarrow \infty} \int_{\Omega_{1}}\left|D_{P}\left(w_{j}\left|b_{j}\right| b_{j}\right)\right| .
$$

LEMMA 3.3 (Approximation of functions in $B V(S)$ ) Let $1 \leqslant q<+\infty$ and let $S$ be a bounded domain in $\mathbb{R}^{2}$ with a Lipschitz continuous boundary, $\partial S$. We further assume that $\gamma \neq \emptyset$ is a finite union of connected open subsets of $\partial S$. Let $v_{0} \in W^{1, q}(S)$ be such that $\nabla v_{0} \in B V(S)$ and let $\bar{v} \in L^{q}(S) \cap B V(S)$. Then there exists a family $\left\{\bar{v}_{\varepsilon}\right\} \subset W^{1, q}(S)$ with $\nabla \bar{v}_{\varepsilon} \in B V(S)$ such that $\bar{v}_{\varepsilon}=v_{0}$ on $\gamma$ for every $\varepsilon>0$, and

$$
\begin{gathered}
\lim _{\varepsilon \rightarrow 0}\left\|\bar{v}_{\varepsilon}-\bar{v}\right\|_{L^{q}(S)}=0 \\
\lim _{\varepsilon \rightarrow 0} \int_{S}\left|D \bar{v}_{\varepsilon}\right|=\int_{S}|D \bar{v}|+\int_{\gamma}\left|\bar{v}-v_{0}\right| .
\end{gathered}
$$

Proof. We define a function $v_{\varepsilon}$ which agrees with $v_{0}$ in a thin strip around $\gamma$ and with $v$ in the remainder of $S$. We then construct $\bar{v}_{\varepsilon}$ by mollifying $v_{\varepsilon}$ while preserving its boundary values.

Since $\partial S$ is Lipschitz continuous, there exists a finite open cover $\left\{U_{i}\right\}_{i=1}^{q}$ of $\bar{\gamma}$ such that

$$
\begin{aligned}
\partial S \cap U_{i} & =Q_{i}\left\{x \in \mathbb{R}^{2}: x_{2}=f_{i}\left(x_{1}\right), x_{1} \in\left(\alpha_{i}, \beta_{i}\right)\right\}, \\
S \cap U_{i} & =Q_{i}\left\{x \in \mathbb{R}^{2}: f_{i}\left(x_{1}\right)-\rho<x_{2}<f_{i}\left(x_{1}\right), x_{1} \in\left(\alpha_{i}, \beta_{i}\right)\right\},
\end{aligned}
$$

where $f_{i}$ is Lipschitz continuous, $\alpha_{i}<\beta_{i}, \rho>0$, and $Q_{i}$ is a rigid motion. Next, we let $\phi_{i} \in$ $\mathcal{C}_{0}^{\infty}\left(U_{i}\right)$ be a partition of unity satisfying $\phi_{i} \geqslant 0$ and $\sum_{i} \phi_{i}=1$ near $\bar{\gamma}$. 
We further assume that

$$
\gamma \cap U_{i}=Q_{i}\left\{x \in \mathbb{R}^{2}: x_{2}=f_{i}\left(x_{1}\right), x_{1} \in\left(\bar{\alpha}_{i}, \bar{\beta}_{i}\right)\right\},
$$

where $\left(\bar{\alpha}_{i}, \bar{\beta}_{i}\right)=\left(\alpha_{i}, \beta_{i}\right)$ unless $Q_{i}\left(\bar{\alpha}_{i}, f_{i}\left(\bar{\alpha}_{i}\right)\right)$ for $\bar{\alpha}_{i} \in\left(\alpha_{i}, \beta_{i}\right)$ is an end point of $\gamma$ or $Q_{i}\left(\bar{\beta}_{i}, f_{i}\left(\bar{\beta}_{i}\right)\right)$ for $\bar{\beta}_{i} \in\left(\alpha_{i}, \beta_{i}\right)$ is an end point of $\gamma$. For $0<\varepsilon<\rho$ and each $i=1, \ldots, q$, we define a strip

$$
E_{i}^{\varepsilon}=Q_{i}\left\{x \in \mathbb{R}^{2}: f_{i}\left(x_{1}\right)-\varepsilon<x_{2}<f_{i}\left(x_{1}\right), x_{1} \in\left(\bar{\alpha}_{i}, \bar{\beta}_{i}\right)\right\},
$$

and we let $\chi_{i}^{\varepsilon}$ denote its characteristic function. We now define

$$
v_{\varepsilon}=\bar{v}-\sum_{i} \phi_{i} \chi_{i}^{\varepsilon}\left(\bar{v}-v_{0}\right)=\left(1-\sum_{i} \phi_{i} \chi_{i}^{\varepsilon}\right) \bar{v}+\sum_{i} \phi_{i} \chi_{i}^{\varepsilon} v_{0} .
$$

We then have that $v_{\varepsilon}=v_{0}$ on $\gamma$, and $v_{\varepsilon} \rightarrow \bar{v}$ in $L^{q}(S)$ as $\varepsilon \rightarrow 0$.

Now

$$
\begin{aligned}
\int_{S}\left|D\left(\phi_{i} \chi_{i}^{\varepsilon}\left(\bar{v}-v_{0}\right)\right)\right|= & \int_{Q_{i}\left\{\left(x_{1}, f_{i}\left(x_{1}\right)-\varepsilon\right): x_{1} \in\left(\bar{\alpha}_{i}, \bar{\beta}_{i}\right)\right\}} \phi_{i}\left|\bar{v}-v_{0}\right|+\int_{E_{i}^{\varepsilon}}\left|D\left[\phi_{i}\left(\bar{v}-v_{0}\right)\right]\right| \\
& +\int_{Q_{i}\left\{\left(\bar{\alpha}_{i}, f_{i}\left(\bar{\alpha}_{i}\right)-s\right): 0<s<\varepsilon\right\}} \phi_{i}\left|\bar{v}-v_{0}\right|+\int_{Q_{i}\left\{\left(\bar{\beta}_{i}, f_{i}\left(\bar{\beta}_{i}\right)-s\right): 0<s<\varepsilon\right\}} \phi_{i}\left|\bar{v}-v_{0}\right| .
\end{aligned}
$$

By general properties of the trace [15], the first term converges to $\int_{Q_{i}\left\{\left(x_{1}, f_{i}\left(x_{1}\right)\right): x_{1} \in\left(\bar{\alpha}_{i}, \bar{\beta}_{i}\right)\right\}} \phi_{i}\left|\bar{v}-v_{0}\right|$ as $\varepsilon \rightarrow 0$, and the second term on the right-hand side converges to zero as $\varepsilon \rightarrow 0$ since the $E_{i}^{\varepsilon}$ are decreasing and $\cap_{\varepsilon} E_{i}^{\varepsilon}=\emptyset$. Since the $Q_{i}\left\{\left(\bar{\alpha}_{i}, f_{i}\left(\bar{\alpha}_{i}\right)-s\right): 0<s<\varepsilon\right\}$ are decreasing as $\varepsilon \rightarrow 0$, and $\cap_{\varepsilon} Q_{i}\left\{\left(\bar{\alpha}_{i}, f_{i}\left(\bar{\alpha}_{i}\right)-s\right): 0<s<\varepsilon\right\}=\emptyset$, we can conclude that the third term on the right-hand side above converges to zero and a similar argument gives the convergence of the final term on the right-hand side to zero. (We note that the third and fourth terms are zero if $\left(\bar{\alpha}_{i}, \bar{\beta}_{i}\right)=\left(\alpha_{i}, \beta_{i}\right)$.) Hence, we can obtain by applying the triangle inequality to (3.7) and using the above inequalities that

$$
\limsup _{\varepsilon \rightarrow 0} \int_{S}\left|D v_{\varepsilon}\right| \leqslant \int_{S}|D \bar{v}|+\int_{\gamma}\left|\bar{v}-v_{0}\right|,
$$

and so by the lower-semicontinuity Lemma 3.2 we have that

$$
\lim _{\varepsilon \rightarrow 0} \int_{S}\left|D v_{\varepsilon}\right|=\int_{S}|D \bar{v}|+\int_{\gamma}\left|\bar{v}-v_{0}\right|
$$

By Theorem 3.3, for each $v_{\varepsilon}$ there exists a sequence $v_{\varepsilon j} \in \mathcal{C}^{\infty}(S)$ such that $v_{\varepsilon j}=v_{\varepsilon}=v_{0}$ on $\gamma$, $\nabla v_{\varepsilon j} \in B V(S)$, and such that

$$
\lim _{j \rightarrow \infty}\left\|v_{\varepsilon j}-v_{\varepsilon}\right\|_{L^{q}(S)}=0 \text { and } \quad \lim _{j \rightarrow \infty} \int_{S}\left|D v_{\varepsilon j}\right|=\int_{S}\left|D v_{\varepsilon}\right| .
$$

We can hence choose $j=j(\varepsilon)$ such that $\bar{v}_{\varepsilon}=v_{\varepsilon j(\varepsilon)}$ satisfies the conclusion of Lemma 3.3.

We can now derive the following theorem that will be used in the derivation of the thin-film limit. 
THEOREM 3.4 Let $1 \leqslant q<+\infty$ and let $S$ be a bounded domain in $\mathbb{R}^{2}$ with a Lipschitz continuous boundary, $\partial S$. We further assume that $\gamma \neq \emptyset$ is a finite union of connected open subsets of $\partial S$. Let $b_{0} \in W^{1, q}(S)$ be such that $\nabla b_{0} \in B V(S)$, let $\bar{b} \in L^{q}(S) \cap B V(S)$, and let $w \in B V(S)$. Then there exists a family $\left\{\bar{b}_{\varepsilon}\right\} \subset W^{1, q}(S)$ with $\nabla \bar{b}_{\varepsilon} \in B V(S)$ such that $\bar{b}_{\varepsilon}=b_{0}$ on $\gamma$ for every $\varepsilon>0$, and

$$
\begin{gathered}
\lim _{\varepsilon \rightarrow 0}\left\|\bar{b}_{\varepsilon}-\bar{b}\right\|_{L^{q}(S)}=0 \\
\lim _{\varepsilon \rightarrow 0} \int_{S}\left|D\left(w\left|\bar{b}_{\varepsilon}\right| \bar{b}_{\varepsilon}\right)\right|=\int_{S}|D(w|\bar{b}| \bar{b})|+\sqrt{2} \int_{\gamma}\left|\bar{b}-b_{0}\right| .
\end{gathered}
$$

Proof. We construct the family $\left\{\bar{b}_{\varepsilon}\right\} \subset W^{1, q}(S)$ exactly as in Lemma 3.3, and the proof of (3.8) is obtained by following the proof of (3.6).

\section{Existence of minimizers for the finite-thickness model}

We have the following lemma establishing the existence of minimizers of the energy (2.5) for all $0<h \leqslant 1$.

LEMMA 4.1 We assume that $S$ is a bounded domain with Lipschitz continuous boundary, $\partial S$, and that $\gamma \neq \emptyset$ is an open subset of $\partial S$. Then there exists $\tilde{u}_{h} \in \mathcal{A}_{h}$ such that

$$
\mathcal{E}_{h}\left(\tilde{u}_{h}\right)=\inf _{\tilde{u} \in \mathcal{A}_{h}} \mathcal{E}_{h}(\tilde{u})
$$

for the energy $\mathcal{E}_{h}$ defined in (2.5) and the space $\mathcal{A}_{h}$ of admissible deformations defined in (2.4).

Proof. Since $\tilde{u}_{0} \in \mathcal{A}_{h}$ and $\mathcal{E}_{h}(\tilde{u}) \geqslant 0$, we can consider an energy-minimizing sequence $\left\{\tilde{u}_{k}\right\} \subset \mathcal{A}_{h}$, that is,

$$
\mathcal{E}_{h}\left(\tilde{u}_{k}\right) \rightarrow \inf _{\tilde{u} \in \mathcal{A}_{h}} \mathcal{E}_{h}(\tilde{u}) \quad \text { as } k \rightarrow \infty .
$$

Using the lower bound on $\phi$ in (2.2) and the Poincaré inequality, together with the boundary conditions for $\tilde{u}_{k}$, we can obtain that there exists a positive constant $C$ such that

$$
\left\|\nabla \tilde{u}_{k}\right\|_{B V\left(\Omega_{h}\right)}+\left\|\tilde{u}_{k}\right\|_{W^{1, p}\left(\Omega_{h}\right)} \leqslant C \text { for all } k .
$$

Hence, it follows from the compactness Theorem 3.2, the semi-continuity Theorem 3.1, and the trace theorem for functions in $W^{1,1}\left(\Omega_{h} ; \mathbb{R}^{3}\right)$ that there exists $\tilde{u}_{h} \in \mathcal{A}_{h}$ and a subsequence of $\left\{\tilde{u}_{k}\right\}$, not relabeled, such that

$$
\tilde{u}_{k} \rightarrow \tilde{u}_{h} \text { in } W^{1, p}\left(\Omega_{h} ; \mathbb{R}^{3}\right) \quad \text { and } \quad \tilde{u}_{k} \rightarrow \tilde{u}_{h} \text { in } W^{1,1}\left(\Omega_{h} ; \mathbb{R}^{3}\right) \quad \text { as } k \rightarrow \infty \text {. }
$$

Since $\phi$ is non-negative and continuous, Fatou's lemma and the semi-continuity Theorem 3.1 give the result

$$
\mathcal{E}_{h}\left(\tilde{u}_{h}\right) \leqslant \liminf _{k \rightarrow \infty} \mathcal{E}_{h}\left(\tilde{u}_{k}\right)=\inf _{\tilde{u} \in \mathcal{A}_{h}} \mathcal{E}_{h}(\tilde{u})
$$




\section{Derivation of the thin-film variational principle}

We next study the behavior of minimizers $\left\{\tilde{u}_{h} \in \mathcal{A}_{h}: 0<h \leqslant 1\right\}$ as the thickness of the film, $h$, converges to zero. We will need the following characterization of $\int_{\Omega}|D(\nabla v)|$ for $v \in W^{1,1}\left(\Omega ; \mathbb{R}^{3}\right)$ with $\nabla v \in B V(\Omega)$ that groups the sums into those involving no derivatives with respect to $x_{3}$, those involving one derivative with respect to $x_{3}$, and those involving two derivatives with respect to $x_{3}$. The proof follows directly from the definition (2.1) by using integration by parts to obtain

$$
\int_{\Omega} v_{i, j} \psi_{i j 3,3}=\int_{\Omega} v_{i, 3} \psi_{i j 3, j}
$$

for $v \in W^{1,1}\left(\Omega ; \mathbb{R}^{3}\right)$ and $\psi_{i j 3} \in \mathcal{C}_{0}^{\infty}(\Omega)$

LEMMA 5.1 Let $\Omega \subset \mathbb{R}^{3}$ be an open set and let $v \in W^{1,1}\left(\Omega ; \mathbb{R}^{3}\right)$ be such that $\nabla v \in B V(\Omega)$. Then

$$
\begin{aligned}
\int_{\Omega}|D(\nabla v)|=\sup & \left\{\sum_{\substack{i=1,2,3 \\
j, k=1,2}} \int_{\Omega} v_{i, j} \psi_{i j k, k}+\sum_{\substack{i=1,2,3 \\
j=1,2}} \int_{\Omega} v_{i, 3} \psi_{i j 3, j}+\sum_{\substack{i=1,2,3 \\
k=1,2}} \int_{\Omega} v_{i, 3} \psi_{i 3 k, k}\right. \\
& \left.+\sum_{i=1,2,3} \int_{\Omega} v_{i, 3} \psi_{i 33,3}: \psi \in \mathcal{C}_{0}^{\infty}(\Omega),|\psi(x)| \leqslant 1 \text { for all } x \in \Omega\right\} .
\end{aligned}
$$

To be able to analyze the thin film limit on a fixed domain, we associate to each deformation $\tilde{u} \in \mathcal{A}_{h}$ a deformation $u: \Omega_{1} \rightarrow \mathbb{R}^{3}$ via

$$
u\left(z_{1}, z_{2}, z_{3}\right)=\tilde{u}\left(z_{1}, z_{2}, h z_{3}\right) \quad \text { for } z=\left(z_{1}, z_{2}, z_{3}\right) \in \Omega_{1}
$$

We note that since $\tilde{u} \in \mathcal{A}_{h}$, we have that

$$
u \in \mathcal{A}_{1}^{(h)} \equiv\left\{v \in W^{1, p}\left(\Omega_{1} ; \mathbb{R}^{3}\right): \nabla v \in B V\left(\Omega_{1}\right), v=u_{0} \text { on } \Gamma_{1}\right\}
$$

where

$$
u_{0}\left(z_{1}, z_{2}, z_{3}\right)=\tilde{u}_{0}\left(z_{1}, z_{2}, h z_{3}\right) \quad \text { for } z=\left(z_{1}, z_{2}, z_{3}\right) \in \Omega_{1}
$$

Since the energy is expected to scale linearly in $h$ as $h \rightarrow 0$, we will consider for each $u \in \mathcal{A}_{1}^{(h)}$ the scaled energy $\mathcal{E}_{1}^{(h)}$ defined by

$$
\mathcal{E}_{1}^{(h)}(u)=\frac{1}{h} \mathcal{E}_{h}(\tilde{u}) .
$$


In view of Lemma 5.1, we can write for $u \in \mathcal{A}_{1}^{(h)}$

$$
\begin{aligned}
\mathcal{E}_{1}^{(h)}(u)= & \kappa \sup \left\{\sum_{\substack{i=1,2,3 \\
j, k=1,2}} \int_{\Omega_{1}} u_{i, j} \psi_{i j k, k} \mathrm{~d} z+\sum_{\substack{i=1,2,3 \\
j=1,2}} \int_{\Omega_{1}} h^{-1} u_{i, 3} \psi_{i j 3, j} \mathrm{~d} z+\sum_{\substack{i=1,2,3 \\
k=1,2}} \int_{\Omega_{1}} h^{-1} u_{i, 3} \psi_{i 3 k, k} \mathrm{~d} z\right. \\
& \left.+\sum_{i=1,2,3} \int_{\Omega_{1}} h^{-2} u_{i, 3} \psi_{i 33,3} \mathrm{~d} z: \psi \in \mathcal{C}_{0}^{\infty}\left(\Omega_{1}\right),|\psi(z)| \leqslant 1 \text { for all } z \in \Omega_{1}\right\} \\
& +\int_{\Omega_{1}} \phi\left(u, 1|u, 2| h^{-1} u, 3\right) \mathrm{d} z,
\end{aligned}
$$

where the subindices denote differentiation with respect to $z \in \Omega_{1}$.

We can now state and prove our main theorem on the thin-film limit of the total-variation model.

THEOREM 5.1 Let $S$ be a bounded domain in $\mathbb{R}^{2}$ with a Lipschitz continuous boundary, $\partial S$, and let $\gamma \neq \emptyset$ be a finite union of connected $\mathcal{C}^{1,1}$ open subsets of $\partial S$. For every subsequence $\left\{u_{h_{n}}\right\}$ with $h_{n} \rightarrow 0$ as $n \rightarrow \infty$ of the family of rescaled minimizers (4.1), $\left\{u_{h} \in \mathcal{A}_{1}^{(h)}: 0<h \leqslant 1\right\}$, there exist a further subsequence, not relabeled, and $(\bar{y}, \bar{b}) \in \mathcal{A}_{0}$ such that if $(\hat{y}(z), \hat{b}(z))=\left(\bar{y}\left(z_{1}, z_{2}\right), \bar{b}\left(z_{1}, z_{2}\right)\right)$ for $z=\left(z_{1}, z_{2}, z_{3}\right) \in \Omega_{1}$, then

$$
\left.\begin{array}{lllll}
u_{h_{n}} \rightarrow \hat{y} & \text { in } W^{1, p}\left(\Omega_{1} ; \mathbb{R}^{3}\right) & \text { and } & h_{n}^{-1} u_{h_{n}, 3} \rightarrow \hat{b} & \text { in } L^{p}\left(\Omega_{1} ; \mathbb{R}^{3}\right) \\
u_{h_{n}} \rightarrow \hat{y} & \text { in } W^{1,1}\left(\Omega_{1} ; \mathbb{R}^{3}\right) & \text { and } & h_{n}^{-1} u_{h_{n}, 3} \rightarrow \hat{b} & \text { in } L^{1}\left(\Omega_{1} ; \mathbb{R}^{3}\right)
\end{array}\right\} \text { as } n \rightarrow \infty .
$$

Furthermore,

$$
\lim _{n \rightarrow \infty} \mathcal{E}_{1}^{\left(h_{n}\right)}\left(u_{h_{n}}\right)=\mathcal{E}^{(0)}(\bar{y}, \bar{b})
$$

where

$$
\mathcal{E}^{(0)}(y, b)=\kappa\left[\int_{S}|D(\nabla y|b| b)|+\sqrt{2} \int_{\gamma}\left|b-b_{0}\right|\right]+\int_{S} \phi(\nabla y \mid b)
$$

for $(y, b) \in \mathcal{A}_{0}=\left\{(y, b) \in W^{1, p}\left(S ; \mathbb{R}^{3}\right) \times L^{p}\left(S ; \mathbb{R}^{3}\right): \nabla y, b \in B V(S), y=y_{0}\right.$ on $\left.\gamma\right\}$ and

$$
\mathcal{E}^{(0)}(\bar{y}, \bar{b})=\min _{(y, b) \in \mathcal{A}_{0}} \mathcal{E}^{(0)}(y, b) .
$$

Proof. We first observe that

$$
\mathcal{E}_{1}^{(h)}\left(u_{h}\right)=\frac{1}{h} \mathcal{E}_{h}\left(\tilde{u}_{h}\right) \leqslant \frac{1}{h} \mathcal{E}_{h}\left(\tilde{u}_{0}\right)=\mathcal{E}_{1}^{(h)}\left(u_{0}\right)
$$

for all $0<h \leqslant 1$ and that $\mathcal{E}_{1}^{(h)}\left(u_{0}\right)$ is a constant independent of $h$. Hence, using the Poincaré 
inequality, we see that there exists a positive constant $C$ such that

$$
\begin{gathered}
\left\|u_{h}\right\|_{W^{1, p}\left(\Omega_{1} ; \mathbb{R}^{3}\right)} \leqslant C, \quad\left\|h^{-1} u_{h, 3}\right\|_{L^{p}\left(\Omega_{1} ; \mathbb{R}^{3}\right)} \leqslant C, \\
\int_{\Omega_{1}}\left|D\left(\nabla u_{h}\right)\right| \leqslant C, \quad \int_{\Omega_{1}}\left|D\left(h^{-1} u_{h, 3}\right)\right| \leqslant C, \\
\sup \left\{\sum_{i=1,2,3} \int_{\Omega_{1}} h^{-2}\left(u_{h}\right)_{i, 3} \psi_{i, 3}: \psi \in \mathcal{C}_{0}^{\infty}\left(\Omega_{1}\right),|\psi(z)| \leqslant 1 \text { for all } z \in \Omega_{1}\right\} \leqslant C
\end{gathered}
$$

for all $0<h \leqslant 1$. It then follows from the compactness Theorem 3.2 and the semi-continuity Theorem 3.1 that there exist $\hat{y} \in W^{1, p}\left(\Omega_{1} ; \mathbb{R}^{3}\right)$ with $\nabla \hat{y} \in B V\left(\Omega_{1}\right)$ and $\hat{b} \in L^{p}\left(\Omega_{1} ; \mathbb{R}^{3}\right)$ with $\hat{b} \in B V\left(\Omega_{1}\right)$ such that for a further subsequence of $\left\{u_{h_{n}}\right\}$, not relabeled, we have that

$$
\left.\begin{array}{lllll}
u_{h_{n}} \rightarrow \hat{y} & \text { in } W^{1, p}\left(\Omega_{1} ; \mathbb{R}^{3}\right) & \text { and } & h_{n}^{-1} u_{h_{n}, 3} \rightarrow \hat{b} & \text { in } L^{p}\left(\Omega_{1} ; \mathbb{R}^{3}\right) \\
u_{h_{n}} \rightarrow \hat{y} & \text { in } W^{1,1}\left(\Omega_{1} ; \mathbb{R}^{3}\right) & \text { and } & h_{n}^{-1} u_{h_{n}, 3} \rightarrow \hat{b} & \text { in } L^{1}\left(\Omega_{1} ; \mathbb{R}^{3}\right)
\end{array}\right\} \text { as } n \rightarrow \infty .
$$

We have by the trace theorem that $\hat{y}=y_{0}$ on $\Gamma_{1}$. Finally, it follows from (5.3) and (5.5) that $\hat{y}$ and $\hat{b}$ are independent of $z_{3}$, so the existence of $(\bar{y}, \bar{b}) \in \mathcal{A}_{0}$ follows.

Next, we have that

$$
\int_{\Omega_{1}}\left|D_{P}\left(\nabla_{P} u_{h}\left|h^{-1} u_{h, 3}\right| h^{-1} u_{h, 3}\right)\right| \leqslant \frac{1}{h} \int_{\Omega_{h}}\left|D\left(\nabla \tilde{u}_{h}\right)\right|
$$

since

$$
\begin{aligned}
& \sup \left\{\sum_{\substack{i=1,2,3 \\
j, k=1,2}} \int_{\Omega_{1}}\left(u_{h}\right)_{i, j} \psi_{i j k, k}+\sum_{\substack{i=1,2,3 \\
j=1,2}} \int_{\Omega_{1}} h^{-1}\left(u_{h}\right)_{i, 3} \psi_{i j 3, j}\right. \\
& \left.\quad+\sum_{\substack{i=1,2,3 \\
k=1,2}} \int_{\Omega_{1}} h^{-1}\left(u_{h}\right)_{i, 3} \psi_{i 3 k, k}: \psi \in \mathcal{C}_{0}^{\infty}\left(\Omega_{1}\right),|\psi(z)| \leqslant 1 \text { for all } z \in \Omega_{1}\right\} \\
& \leqslant \sup \left\{\sum_{\substack{i=1,2,3 \\
j, k=1,2}} \int_{\Omega_{1}}\left(u_{h}\right)_{i, j} \psi_{i j k, k}+\sum_{\substack{i=1,2,3 \\
j=1,2}} \int_{\Omega_{1}} h^{-1}\left(u_{h}\right)_{i, 3} \psi_{i j 3, j}+\sum_{i=1,2,3} \int_{\Omega_{1}} h^{-1}\left(u_{h}\right)_{i, 3} \psi_{i 3 k, k}\right. \\
& \left.\quad+\sum_{i=1,2,3} \int_{\Omega_{1}} h^{-2}\left(u_{h}\right)_{i, 3} \psi_{i 33,3}: \psi \in \mathcal{C}_{0}^{\infty}\left(\Omega_{1}\right),|\psi(z)| \leqslant 1 \text { for all } z \in \Omega_{1}\right\}
\end{aligned}
$$

Since $\nabla_{P} u_{h_{n}} \rightarrow \nabla_{P} \hat{y}$ in $L^{1}\left(\Omega_{1} ; \mathbb{R}^{3 \times 2}\right)$ and $h^{-1} u_{h_{n}, 3} \rightarrow \hat{b}$ in $L^{1}\left(\Omega_{1} ; \mathbb{R}^{3}\right)$, a further subsequence converges almost everywhere in $\Omega_{1}$; it then follows from (5.7), Corollary 3.1, and Fatou's lemma that since the trace of $h^{-1} u_{h, 3}$ on $\Gamma_{1}$ is $b_{0}$ for all $0<h \leqslant 1$ we have that

$$
\mathcal{E}^{(0)}(\bar{y}, \bar{b})=\kappa\left[\int_{\Omega_{1}}\left|D_{P}\left(\nabla_{P} \hat{y}|\hat{b}| \hat{b}\right)\right|+\sqrt{2} \int_{\Gamma_{1}}\left|\hat{b}-b_{0}\right|\right]+\int_{\Omega_{1}} \phi\left(\nabla_{P} \hat{y} \mid \hat{b}\right) \leqslant \liminf _{n \rightarrow \infty} \mathcal{E}_{1}^{\left(h_{n}\right)}\left(u_{h_{n}}\right) .
$$


To prove (5.1), we will construct a sequence of admissible deformations on $\Omega_{h}$ such that the rescaled energies of the associated deformations on $\Omega_{1}$ converge to the left-hand side of (5.8). The existence of such a sequence will establish equality in (5.8) with 'lim' instead of 'lim inf' and thus prove (5.1).

Since $b_{0} \in W^{1, p}\left(S ; \mathbb{R}^{3}\right)$ and $\nabla b_{0} \in B V(S)$, we can use Theorem 3.4 to obtain a sequence of functions $\bar{b}_{\varepsilon} \in W^{1, p}(S)$ with $\nabla \bar{b}_{\varepsilon} \in B V(S)$ such that $\bar{b}_{\varepsilon}=b_{0}$ on $\gamma$ for every $\varepsilon>0$, and

$$
\begin{gathered}
\lim _{\varepsilon \rightarrow 0}\left\|\bar{b}_{\varepsilon}-\bar{b}\right\|_{L^{p}(S)}=0, \\
\lim _{\varepsilon \rightarrow 0} \int_{S}\left|D\left(\nabla \bar{y}\left|\bar{b}_{\varepsilon}\right| \bar{b}_{\varepsilon}\right)\right|=\int_{S}|D(\nabla \bar{y}|\bar{b}| \bar{b})|+\sqrt{2} \int_{\gamma}\left|\bar{b}-b_{0}\right| .
\end{gathered}
$$

Consider now the test functions

$$
\bar{u}_{h}^{\varepsilon}\left(z_{1}, z_{2}, z_{3}\right)=\bar{y}\left(z_{1}, z_{2}\right)+h z_{3} \bar{b}_{\varepsilon}\left(z_{1}, z_{2}\right) \in \mathcal{A}_{1}^{(h)} .
$$

Since $\nabla_{P} \bar{u}_{h}^{\varepsilon}=\nabla_{P} \bar{y}+h z_{3} \nabla_{P} \bar{b}_{\varepsilon} \rightarrow \nabla_{P} \bar{y}$ in $L^{p}\left(\Omega_{1}\right)$ as $h \rightarrow 0$, we have

$$
\int_{\Omega_{1}} \phi\left(\nabla \bar{u}_{h}^{\varepsilon}\right)=\int_{\Omega_{1}} \phi\left(\nabla_{P} \bar{u}_{h}^{\varepsilon} \mid \bar{b}_{\varepsilon}\right) \rightarrow \int_{\Omega_{1}} \phi\left(\nabla_{P} \bar{y} \mid \bar{b}_{\varepsilon}\right)=\int_{S} \phi\left(\nabla \bar{y} \mid \bar{b}_{\varepsilon}\right) \quad \text { as } h \rightarrow 0,
$$

using the growth condition on $\phi$ and the dominated convergence theorem. Similarly,

$$
\int_{S} \phi\left(\nabla \bar{y} \mid \bar{b}_{\varepsilon}\right) \rightarrow \int_{S} \phi(\nabla \bar{y} \mid \bar{b}) \quad \text { as } \varepsilon \rightarrow 0,
$$

so

$$
\lim _{\varepsilon \rightarrow 0} \lim _{h \rightarrow 0} \int_{\Omega_{1}} \phi\left(\nabla \bar{u}_{h}^{\varepsilon}\right)=\int_{S} \phi(\nabla \bar{y} \mid \bar{b}) .
$$

Since $\bar{b}_{\varepsilon}$ is independent of $z_{3}$, we have that

$$
\int_{\Omega_{1}}\left|D\left(\nabla \bar{u}_{h}^{\varepsilon}\right)\right|=\int_{\Omega_{1}}\left|D_{P}\left(\nabla_{P}\left(\bar{u}_{h}^{\varepsilon}\right)\left|\bar{b}_{\varepsilon}\right| \bar{b}_{\varepsilon}\right)\right|=\int_{\Omega_{1}}\left|D_{P}\left(\nabla_{P}\left(\bar{y}+h z_{3} \bar{b}_{\varepsilon}\right)\left|\bar{b}_{\varepsilon}\right| \bar{b}_{\varepsilon}\right)\right|
$$

because

$$
\begin{gathered}
\sup \left\{\sum_{\substack{i=1,2,3 \\
j, k=1,2}} \int_{\Omega_{1}}\left(\bar{u}_{h}^{\varepsilon}\right)_{i, j} \psi_{i j k, k}+\sum_{\substack{i=1,2,3 \\
j=1,2}} \int_{\Omega_{1}} h^{-1}\left(\bar{u}_{h}^{\varepsilon}\right)_{i, 3} \psi_{i j 3, j}+\sum_{\substack{i=1,2,3 \\
k=1,2}} \int_{\Omega_{1}} h^{-1}\left(\bar{u}_{h}^{\varepsilon}\right)_{i, 3} \psi_{i 3 k, k}\right. \\
\left.\quad+\sum_{i=1,2,3} \int_{\Omega_{1}} h^{-2}\left(\bar{u}_{h}^{\varepsilon}\right)_{i, 3} \psi_{i 33,3}: \psi \in \mathcal{C}_{0}^{\infty}(\Omega),|\psi(z)| \leqslant 1 \text { for all } z \in \Omega_{1}\right\} \\
=\sup \left\{\sum_{\substack{i=1,2,3 \\
j, k=1,2}} \int_{\Omega_{1}}\left(\bar{u}_{h}^{\varepsilon}\right)_{i, j} \psi_{i j k, k}+\sum_{\substack{i=1,2,3 \\
j=1,2}} \int_{\Omega_{1}} \bar{b}_{\varepsilon} \psi_{i j 3, j}\right. \\
\left.+\sum_{\substack{i=1,2,3 \\
k=1,2}} \int_{\Omega_{1}} \bar{b}_{\varepsilon} \psi_{i 3 k, k}: \psi \in \mathcal{C}_{0}^{\infty}\left(\Omega_{1}\right),|\psi(z)| \leqslant 1 \text { for all } z \in \Omega_{1}\right\}
\end{gathered}
$$


We have by the triangle inequality that

$$
\begin{aligned}
\int_{\Omega_{1}}\left|D_{P}\left(\nabla_{P} \bar{y}\left|\bar{b}_{\varepsilon}\right| \bar{b}_{\varepsilon}\right)\right|-\int_{\Omega_{1}}\left|D_{P}\left(h z_{3} \nabla_{P} \bar{b}_{\varepsilon}\right)\right| & \leqslant \int_{\Omega_{1}}\left|D_{P}\left(\nabla_{P}\left(\bar{y}+h z_{3} \bar{b}_{\varepsilon}\right)\left|\bar{b}_{\varepsilon}\right| \bar{b}_{\varepsilon}\right)\right| \\
& \leqslant \int_{\Omega_{1}}\left|D_{P}\left(\nabla_{P} \bar{y}\left|\bar{b}_{\varepsilon}\right| \bar{b}_{\varepsilon}\right)\right|+\int_{\Omega_{1}}\left|D_{P}\left(h z_{3} \nabla_{P} \bar{b}_{\varepsilon}\right)\right|,
\end{aligned}
$$

so we have since $\nabla \bar{b}_{\varepsilon} \in B V(S)$ and $\bar{y}$ and $\bar{b}_{\varepsilon}$ are independent of $z_{3}$ that

$$
\lim _{h \rightarrow 0} \int_{\Omega_{1}}\left|D\left(\nabla \bar{u}_{h}^{\varepsilon}\right)\right|=\lim _{h \rightarrow 0} \int_{\Omega_{1}}\left|D_{P}\left(\nabla_{P}\left(\bar{y}+h z_{3} \bar{b}_{\varepsilon}\right)\left|\bar{b}_{\varepsilon}\right| \bar{b}_{\varepsilon}\right)\right|=\int_{S}\left|D\left(\nabla \bar{y}\left|\bar{b}_{\varepsilon}\right| \bar{b}_{\varepsilon}\right)\right| .
$$

We thus have from (5.9) and (5.11) that

$$
\lim _{\varepsilon \rightarrow 0} \lim _{h \rightarrow 0} \int_{\Omega_{1}}\left|D\left(\nabla \bar{u}_{h}^{\varepsilon}\right)\right|=\int_{S}|D(\nabla \bar{y}|\bar{b}| \bar{b})|+\sqrt{2} \int_{\gamma}\left|\bar{b}-b_{0}\right|,
$$

and from (5.10) and the above identity (5.12) we have that

$$
\lim _{\varepsilon \rightarrow 0} \lim _{h \rightarrow 0} \mathcal{E}_{1}^{(h)}\left(\bar{u}_{h}^{\varepsilon}\right)=\mathcal{E}^{(0)}(\bar{y}, \bar{b})
$$

for $\mathcal{E}^{(0)}$ defined in (5.2). Since $u_{h}$ is a minimizing deformation for $\mathcal{E}_{1}^{(h)}$, we have that

$$
\lim _{\varepsilon \rightarrow 0} \lim _{h \rightarrow 0} \mathcal{E}_{1}^{(h)}\left(\bar{u}_{h}^{\varepsilon}\right) \geqslant \limsup _{h \rightarrow 0} \mathcal{E}_{1}^{(h)}\left(u_{h}\right) .
$$

It thus follows from (5.8) and (5.13) that

$$
\lim _{n \rightarrow \infty} \mathcal{E}_{1}^{\left(h_{n}\right)}\left(u_{h_{n}}\right)=\mathcal{E}^{(0)}(\bar{y}, \bar{b})
$$

for any subsequence $\left\{u_{h_{n}}\right\}$ satisfying the limits (5.6).

If we repeat the above argument with an arbitrary pair $(y, b) \in W^{1, p}\left(S ; \mathbb{R}^{3}\right) \times L^{p}\left(S ; \mathbb{R}^{3}\right)$ such that $\nabla y, b \in B V(S)$ and $y=y_{0}$ on $\Gamma_{1}$, we obtain the minimum principle

$$
\mathcal{E}^{(0)}(\bar{y}, \bar{b})=\min _{(y, b) \in \mathcal{A}_{0}} \mathcal{E}^{(0)}(y, b) .
$$

To see this, we can obtain from Theorem 3.4 a sequence of functions $b_{\varepsilon} \in W^{1, p}(S)$ with $\nabla b_{\varepsilon} \in$ $B V(S)$ such that $b_{\varepsilon}=b_{0}$ on $\gamma$ for every $\varepsilon>0$, and

$$
\begin{gathered}
\lim _{\varepsilon \rightarrow 0}\left\|b_{\varepsilon}-b\right\|_{L^{p}(S)}=0, \\
\lim _{\varepsilon \rightarrow 0} \int_{S}\left|D\left(\nabla y\left|b_{\varepsilon}\right| b_{\varepsilon}\right)\right|=\int_{S}|D(\nabla y|b| b)|+\sqrt{2} \int_{\gamma}\left|b-b_{0}\right| .
\end{gathered}
$$

Consider now the test functions

$$
u_{h}^{\varepsilon}\left(z_{1}, z_{2}, z_{3}\right)=y\left(z_{1}, z_{2}\right)+h z_{3} b_{\varepsilon}\left(z_{1}, z_{2}\right) \in \mathcal{A}_{1}^{(h)} .
$$


Following the proof above, we can obtain that

$$
\lim _{\varepsilon \rightarrow 0} \lim _{h \rightarrow 0} \mathcal{E}_{1}^{(h)}\left(u_{h}^{\varepsilon}\right)=\mathcal{E}^{(0)}(y, b) .
$$

Since $u_{h_{n}}$ is a minimizing deformation for $\mathcal{E}_{1}^{\left(h_{n}\right)}$, we have that

$$
\mathcal{E}^{(0)}(y, b)=\lim _{\varepsilon \rightarrow 0} \lim _{n \rightarrow \infty} \mathcal{E}_{1}^{\left(h_{n}\right)}\left(u_{h_{n}}^{\varepsilon}\right) \geqslant \lim _{n \rightarrow \infty} \mathcal{E}_{1}^{\left(h_{n}\right)}\left(u_{h_{n}}\right)=\mathcal{E}^{(0)}(\bar{y}, \bar{b}) .
$$

Since the subsequence $\left\{u_{h_{n}}\right\}$ in the above Theorem 5.1 was arbitrary, we can conclude the following corollary.

COROLLARY 5.1 We have that

$$
\lim _{h \rightarrow 0} \min _{\tilde{v} \in \mathcal{A}_{h}}\left(\frac{1}{h} \mathcal{E}_{h}(\tilde{v})\right)=\min _{(y, b) \in \mathcal{A}_{0}} \mathcal{E}^{(0)}(y, b) .
$$

REMARK 5.1 We note that it follows from the proof of the above theorem that

$$
\frac{1}{h_{n}} \int_{\Omega_{h_{n}}}\left|D\left(\nabla \tilde{u}_{h_{n}}\right)\right| \rightarrow \int_{S}|D(\nabla \bar{y}|\bar{b}| \bar{b})|+\sqrt{2} \int_{\gamma}\left|\bar{b}-b_{0}\right|
$$

and

$$
\frac{1}{h_{n}} \int_{\Omega_{h_{n}}} \phi\left(\tilde{u}_{h_{n}, 1}\left|\tilde{u}_{h_{n}, 2}\right| \tilde{u}_{h_{n}, 3}\right) \mathrm{d} x \rightarrow \int_{S} \phi(\nabla \bar{y} \mid \bar{b}) \mathrm{d} z_{1} \mathrm{~d} z_{2}
$$

as $h_{n} \rightarrow 0$.

REMARK 5.2 We remark that if $(y, b) \in W^{2,1}\left(S ; \mathbb{R}^{3}\right) \times W^{1,1}\left(S ; \mathbb{R}^{3}\right)$, then

$$
\int_{S}|D(\nabla y|b| b)|=\int_{S}\left(\left|\nabla^{2} y\right|^{2}+2|\nabla b|^{2}\right)^{1 / 2} .
$$

This is similar to the Bhattacharya-James model [5], in which the interfacial energy is modeled by

$$
\int_{S}\left(\left|\nabla^{2} y\right|^{2}+2|\nabla b|^{2}\right)
$$

We also note that the requirement that $b$ be in $H^{1}\left(S ; \mathbb{R}^{3}\right)$ in the Bhattacharya-James model makes the trace of $b$ continuous across smooth curves, while the fact that $b$ is in $B V(S)$ in our model allows the trace of $b$ to jump across interfaces and, in particular, on the boundary, $\partial S$. Since the deformation is modeled by $b_{0}$ outside $\gamma$, this generates an additional term in the interfacial energy given by $\sqrt{2} \kappa \int_{\gamma}\left|b-b_{0}\right|$. However, since $\kappa$ is assumed to be small, this allows the trace of $b$ on $\gamma$ to differ from $b_{0}$ significantly without generating an excessive amount of energy. 


\section{The finite-element approximation}

We now give a brief description of the continuous, piecewise linear finite-element approximation of the total-variation thin-film model. Since most deformations observed in martensitic alloys consist of laminated microstructures in which regions of nearly constant deformation gradient are separated by nearly planar interfaces, the combination of the continuous piecewise linear $\left(\mathcal{P}_{1}\right)$ element for $y$ and the piecewise constant $\left(\mathcal{P}_{0}\right)$ element for $b$ should be an efficient choice. The variation of such functions is zero on each element of a given triangulation; hence, all of the variation is concentrated along the edges of the triangulation.

In what follows, we assume that $S$ is a polygonal domain in $\mathbb{R}^{2}$ and that $\tau$ is a fixed triangulation of $S[7,11]$. The elements of the triangulation $\tau$ are denoted by $K$ and the inter-element edges by $e$. To distinguish between the internal edges of $\tau$ and the boundary edges, we write $e \in S$ for the internal edges and $e \in \partial S$ for the boundary edges.

Given an internal edge $e \in S$ and two elements $K_{1}, K_{2} \in \tau$ sharing the edge $e$, we define the jump across the edge $e$ of a function $f \in B V\left(K_{1} \cup K_{2}\right)$ by

$$
\llbracket f \rrbracket_{e}=f_{e, K_{1}}-f_{e, K_{2}}
$$

where $f_{e, K_{i}}$ denotes the trace on $e$ of $\left.f\right|_{K_{i}}$ for $i=1,2$. Since only the Euclidean norm of the jump will enter the discrete energy below, the sign ambiguity in the definition of the jump will not cause an ambiguity in the description of the energy below. For a boundary edge $e \in \partial S$ that is an edge of an element $K \in \tau$, we define $\left.f\right|_{e}$ to be the trace on $e$ of a function $f \in B V(K)$. Finally, we denote by $\mathcal{P}_{1}(\tau)$ the space of continuous piecewise linear functions on $S$ which are linear on each $K \in \tau$, and by $\mathcal{P}_{0}(\tau)$ the space of piecewise constant functions on $S$ which are constant on each $K \in \tau$. Then for $(y, b) \in \mathcal{P}_{1}(\tau) \times \mathcal{P}_{0}(\tau)$, the energy (5.2) is well defined, and we have

$$
\mathcal{E}^{(0)}(y, b)=\kappa\left(\sum_{e \in S}\left|\llbracket(\nabla y|b| b) \rrbracket_{e}\right||e|+\sqrt{2} \sum_{e \in \gamma}|b|_{e}-\left.b_{0}\right|_{e}|| e \mid\right)+\sum_{K \in \tau} \phi\left(\left.(\nabla y \mid b)\right|_{K}\right)|K|,
$$

where $|e|$ denotes the length of the edge $e,|\cdot|$ denotes the Euclidean vector norm, $|K|$ is the area of the element $K$, and

$$
\left|\llbracket(\nabla y|b| b) \rrbracket_{e}\right|=\left(\left|\llbracket \nabla y \rrbracket_{e}\right|^{2}+2\left|\llbracket b \rrbracket_{e}\right|^{2}\right)^{1 / 2} .
$$

We note that this is a conforming approximation since

$$
\mathcal{P}_{1}(\tau) \times \mathcal{P}_{0}(\tau) \subset\left\{(y, b) \in W^{1, p}\left(S ; \mathbb{R}^{3}\right) \times L^{p}\left(S ; \mathbb{R}^{3}\right): \nabla y, b \in B V(S)\right\} .
$$

Since the above term is not differentiable everywhere, we have regularized it in our numerical simulations [10].

We have proven that the error for the deformation and the local volume fractions of deformation gradients converge to zero as the mesh size converges to zero for the finite element approximation of laminated microstructure for bulk (three-dimensional) geometrically nonlinear models of martensitic crystals $[6,9,14,23-25]$. The total variation of the deformation gradient will generally not converge, even with the addition to the total energy of a surface energy proportional to the total variation of the deformation gradient, unless the mesh is aligned with the microstructure. More details on the approximation properties and implementation of the finite element approximation of our total variation thin film model are given in [10]. 


\section{Acknowledgements}

This work was supported in part by NSF DMS 95-05077, by NSF DMS-00-74043, by AFOSR F49620-98-1-0433, by ARO DAAG55-98-1-0335, by the Institute for Mathematics and its Applications, and by the Minnesota Supercomputer Institute.

\section{REFERENCES}

1. Acerbi, E., Buttazzo, G., \& Percivale, D. A variational definition for the strain energy of an elastic string. J. Elast. 25, (1991) 137-148.

2. ADAMS, R. Sobolev Spaces. Academic, New York (1975).

3. AnZelloti, G., Baldo, S., \& Percivale, D. Dimension reduction in variation problems, asymptotic development in $\gamma$-convergence and thin structures in elasticity. Asymptotic Anal. 9, (1994) 61-100.

4. Ball, J. M. \& James, R. D. Fine phase mixtures as minimizers of energy. Arch. Ration. Mech. Anal. 100, (1987) 13-52.

5. Bhattacharya, K. \& James, R. D. A theory of thin films of martensitic materials with applications to microactuators. J. Mech. Phys. Solids 47, (1999) 531-576.

6. BhatTACHARYA, K., Li, B., \& LUSKIN, M. The simply laminated microstructure in martensitic crystals that undergo a cubic to orthorhombic phase transformation. Arch. Ration. Mech. Anal. 149, (1999) 123154.

7. Brenner, S. C. \& Scott, L. R. The Mathematical Theory of Finite Element Methods. Springer, New York (1994).

8. BĚLÍK, P., BRULE, T., \& Luskin, M. On the numerical modeling of deformations of pressurized martensitic thin films. Math. Modelling Numer. Anal. 35, (2001) 525-548.

9. BĚLíK, P. \& LUSKIN, M. Stability of microstructure for tetragonal to monoclinic martensitic transformations. Math. Modelling Numer. Anal. 34, (2000) 663-685.

10. BĚLík, P. \& LUSKIN, M. A computational model for the indentation and phase transformation of a martensitic thin film. Preprint, (2001).

11. Ciarlet, P. G. The Finite Element Method for Elliptic Problems. North-Holland, Amsterdam (1978).

12. Dong, J. W., Chen, L. C., Palmstrøm, C. J., James, R. D., \& McKernan, S. Molecular beam epitaxy growth of ferromagnetic single crystal (001) $\mathrm{Ni}_{2} \mathrm{MnGa}$ on (001) GaAs. Appl. Phys. Lett. $\mathbf{7 5}$, (1999) 1443-1445.

13. Le Dret, H. \& Raoult, A. The nonlinear membrane model as variational limit of nonlinear threedimensional elasticity. J. Math. Pure Appl. 73, (1995) 549-578.

14. Efendiev, Y. \& LUSKIN, M. Stability of microstructures for some martensitic transformations. Math. Comput. Modelling, to appear.

15. Evans, L. C. \& GARIEPY, R. F. Measure Theory and Fine Properties of Functions. CRC Press, Boca Raton, FL (1992).

16. FOnSECA, I. \& FRANCFORT, G. 3d-2d asymptotic analysis of an optimal design problem for thin films. J. Reine Angew. Math. 505, (1998) 173-202.

17. FonseCA, I. Phase transitions of elastic solid materials. Arch. Ration. Mech. Anal. 107, (1989) 195-223.

18. GIUSTI, E. Minimal Surfaces and Functions of Bounded Variation. Birkhäuser, Basel (1984).

19. KoHn, R. \& Müller, S. Branching of twins near an austenite-twinned-martensite interface. Phil. Mag. A 66, (1992a) 697-715.

20. KoHN, R. \& MüLlER, S. Relaxation and regularization of nonconvex variational problems. In Proceedings of the Second International Conference on Partial Differential Equations, (Rendiconti del Seminario Matematico e Fisico di Milano 62). pp. 89-113. (1992b).

21. Kohn, R. \& MüLler, S. Surface energy and microstructure in coherent phase transitions. Comm. Pure Appl. Math. 47, (1994) 405-435. 
22. Krulevitch, P., Lee, A. P., Ramsey, P. B., Trevino, J. C., Hamilton, J., \& Northrup, M. A. Thin film shape memory microactuators. J. MEMS 5, (1996) 270-282.

23. LI, B. \& LUSKIN, M. Finite element analysis of microstructure for the cubic to tetragonal transformation. SIAM J. Numer. Anal. 35, (1998) 376-392.

24. LUSKIN, M. Approximation of a laminated microstructure for a rotationally invariant, double well energy density. Numer. Math. 75, (1996a) 205-221.

25. Luskin, M. On the computation of crystalline microstructure. Acta Numer. 5, (1996b) 191-257.

26. ModicA, L. \& Mortola, S. Il limite nella $\Gamma$-convergenza di una famiglia di funzionali ellittici. Boll. Un. Mat. Ital. A (5) 14, (1977a) 526-529.

27. Modica, L. \& Mortola, S. Un esempio di $\Gamma$-convergenza. Boll. Un. Mat. Ital. B (5) 14, (1977b) 285-299.

28. MÜlLER, S. Singular perturbations as a selection criterion for periodic minimizing sequences. Calc. Var. Part. Diff. Equ. 1, (1993) 169-204. 